Regular Article

pISSN: 2288-9744, eISSN: 2288-9752

Journal of Forest and Environmental Science

Vol. 30, No. 2, pp. 243-252, May, 2014

http://dx.doi.org/10.7747/JFS.2014.30.2.243

\title{
JEES
}

\section{Enhancing Production of Terpenoids in Metabolically Engineered Transgenic Spearmint (Mentha spicata L.) by Salt and Fungal Elicitors}

\author{
Myung Suk Choi ${ }^{1, \#}$, Dong Jin Park, ${ }^{1, \#}$, Hyun Jin Song, ${ }^{1, \#}$, Ji Yun Min², Seung Mi Kang ${ }^{3}$, Chong Kyu Lee, \\ Kye Man $\mathrm{Cho}^{5}$, Chandrakant Karigar ${ }^{6}$, Ho Kyoung $\mathrm{Kim}^{7}$ and Young Min Kang,* \\ ${ }^{1}$ Department of Forest Environmental Resources E Institute of Agriculture and Life Sciences, Gyeongsang National University, Jinju 660-701, \\ Republic of Korea \\ ${ }^{2}$ Sancheong Oriental Medicinal Herb Institute, Sancheong 666-831, Republic of Korea \\ ${ }^{3}$ Department of Forest Research, Gyeongsangnam-do Forest Environment Research Institute, Jinju 660-871, Republic of Korea \\ ${ }^{4}$ Department of Forest Resources, Gyeongnam National University of Science and Technology, Jinju 660-758, Republic of Korea \\ ${ }^{5}$ Department of Food Science, Gyeongnam National University of Science and Technology, Jinju 660-758, Republic of Korea \\ ${ }^{6}$ Department of Studies $E$ Research in Biochemistry, Tumkur University, Tumkur 572-103, India \\ ${ }^{7}$ Herbal Medicine Resources Group, Herbal Medicine Research Division, Korea Institute of Oriental Medicine (KIOM), Daejeon 305-811, Republic \\ of Korea
}

\begin{abstract}
Forest tree species usually takes for long periods to be harvested and cultivated but spearmints are a good model system for woody plant because of reducing and shortening cultivation time. Spearmints are good model plants (Mentha species) for research about terpenoids production and industrial essential oil manufacture. Isopentenyl pyrophosphate isomerase (Iso) and limonene synthase (Limo) are the key enzymes of terpenoid biosynthesis pathway. Transgenic and wild spearmints (Mentha spicata, MS) were cultured in vitro and assessed for the essential oil contents. The content of essential oil of transgenic spearmint also was enhanced slightly depending on the target terpenoid genes. In an attempt to increase productivity of terpenoids further, salt and fungal elicitation strategy was adopted on transgenic Mentha spicata. The salt $(800 \mathrm{mM} \mathrm{NaCl})$ as abiotic and two fungi (Botrytis cinerea and Glomerella cingulata) as biotic were used for elicitors. In the absence of salt stress four terpenoids were detected from the spearmint extracts, all of them being monoterpenes. On the other hand, the transgenic (MSIso) extracts contained eleven terpenoids (10 monoterpenes and 1 phenylpropene) while transgenic (MSLimo) extracts contained seven monoterpenes. After 3 days of fungal infection, the resistance indices further increased to 4.38, 3.89 and 2.04 for wild type, MSIso and MSLimo, respectively. The salt and fungal elicitators proved beneficial towards modifying both the terpenoids profile and improvement in the composition of essential oil. These results have important applications for the large-scale production of essential oils and forest biotechnology with respect to spearmint.
\end{abstract}

Key Words: Essential oils, Elicitors, Forest biotechnology, Metabolically engineered transgenic spearmint, Terpenoids

Received: October 28, 2013. Revised: January 8, 2014. Accepted: January 8, 2014.

Corresponding author: Young Min Kang

Herbal Medicine Resources Group, Herbal Medicine Research Division, Korea Institute of Oriental Medicine (KIOM), Daejeon, Republic of Korea Tel: 82-42-868-9684, Fax: 82-42-863-9434, E-mail: ymkang@kiom.re.kr \& kang2082@gmail.com

"These authors were contributed equally to this work (co-first authors). 


\section{Introduction}

Forest tree species usually takes for long periods to be cultured but spearmints are a good model system for woody plant because of saving cultivation time. Spearmints are good model plants (Mentha species) for the study about terpenoids production and industrial essential oil manufacture because spearmints have the specific terpenoids biosynthesis pathway and require a short period to grow and harvest (Kang et al. 2012). Spearmint (Mentha spicata, MS) has been most widely cultivated in many countries of East Asia, Europe, America and Australia to meet the requirement of essential oils. Essential oil derived from Mentha species in general is a mixture of terpenoids in various proportions. Spearmint essential oil in particular finds application in flavoring of pharmaceuticals and oral medicinal preparations.

Spearmint is one of the main sources for aroma compounds such as; menthol, carvone, linalool, and linayl acetate used widely in food, cosmetic, flavour, and pharmaceutical industries (Singh et al. 1999). The world market for spearmint oils is approximately 1,500,000 kg/yr and increasing at approximately $5 \% / \mathrm{yr}$. The spearmint oil market, while smaller than peppermint oil (approx. 3,500,000 $\mathrm{kg} / \mathrm{yr}$ ), but is substantial and is one of the larger essential oil commodities (Bienvenu et al. 1999).

Terpenoids in plants are synthesized via the methylerythritol phosphate (MEP) pathway and non-MEP pathway, and structurally are classified as mono-, di-, and sesquiterpenoids. Like all secondary metabolites, terpenoids have also critical functions in plant defense mechanism. The instances of metabolic engineering in essential oils about terpenoids are being reported. The essential oil yield of peppermint was enhanced by the overexpression of 1-deoxy-D-xylulose 5-phosphate reductoisomerase, a MEP pathway gene (Lange et al. 2011). However, metabolic engineering of spearmint for altering terpenoid metabolites is less known.

Plant metabolism can be described as a complex network of proteins and their interacting partners. Thus, the plant metabolism is divided into primary and secondary metabolism by conventional biochemists. Generally the products of primary metabolism are considered the ones essential for plant growth and development (Bryant et al. 1991;
Tegelberg and Julkunen-Tiitto 2001; Laitinen et al. 2004). On the other hand secondary metabolites are considered as the overflow-compounds (Seigler 1998). The nature of secondary compounds specific for certain plant species or tissues or they may be produced only at a certain developmental stage depending on the plant age and the prevailing environmental factors (Bryant and Julkunen-Tittto 1995; Tegelberg et al. 2001; Laitinen et al. 2002). Many secondary metabolites are also inducible as a response to wounding or pathogens (Waterman and Mole 1994; Julkunen-Tiitto et al. 1995). B. cinerea (Gray mold) and $G$. cingulata (Anthracnose) were used as fungal elicitors to improve secondary metabolites and fungal pathogens test for defense mechanism in plants (Jung et al. 2003; Lee et al. 2012; Choi et al. 2012).

Plant cell and tissue cultures for the production of secondary metabolites have met with limited success due to their low yields (Bryant et al. 1991). In order to improve the yield of such products, various techniques have been tried. Among the strategies employed to enhance productivity, an efficient method is the use of biotic and abiotic elicitors. The elicitation of plant cells and tissue can lead to increased yields and can shorten the fermentation time. Since the biosynthesis of secondary metabolites in plants is tightly controlled during development, the metabolites are accumulated by plants in response to stress. Thus, microbial attack is a good material to study defense response in vitro cultures. Although a number of studies have focused on elicitation to increase several metabolites, the mechanism of salt and fungal elicitors at both physical and molecular level has not yet been elucidated.

In our previously published paper, Kang et al. (2012) have introduced two genes specifying the isopentenyl pyrophosphate isomerase (Iso) and Limonene synthase (Limo) of the terpenoid biosynthesis pathway to enhance essential oil components and yields. The aims of this study were to investigate the production of terpenoids by the introduction abiotic and biotic elicitors into the environment of transgenic spearmint. Our hypothesis was that the terpenoids productivity could be enhanced by the optimal formulation of exposure time, salt concentration, and selection of fungal strains. 


\section{Materials and Methods}

\section{Plant materials and construction of gene ex- pression vectors}

Spearmint in vitro seedlings from sterilized seeds were prepared for general tissue culture and genetic transformation. The seeds of spearmint were provided by Agricultural farm of Gyeongsang National University. Seeds were sterilized for $15 \mathrm{~min}$ in $3 \%$ (v/v) $\mathrm{NaClO}$ and rinsed five times with sterile distilled water. The seed were placed in Petri dishes containing $20 \mathrm{~mL}$ of MS solid medium, supplemented with $0.1 \mathrm{mg} / \mathrm{l} \mathrm{GA}_{3}$ (w/v), $30 \%$ sucrose $(\mathrm{w} / \mathrm{v})$ and $0.4 \%$ gelrite $(\mathrm{w} / \mathrm{v})$. The $\mathrm{pH}$ of basal medium was adjusted to 5.7 before autoclaving at $121^{\circ} \mathrm{C}$ for $15 \mathrm{~min}$. The dishes were kept in dark at $25 \pm 1^{\circ} \mathrm{C}$ until the seeds germinated. As well described background in previously publications (Park 2008; Kang et al. 2012), Isoprene synthase isolated from hybrid poplar (Populous alba X P. tremula) and Limonene synthase isolated from lemon (Citrus limon) were cloned into pBI121, which were a gift from Dr. Kim from the Gyeongsang National University. The Isoprene synthase and Limonene synthase cDNA in pBI121 were then cut with the Xba I and Sal I restriction enzymes to release the Isoprene synthase and Limonene synthase gene. Specific information about DNA manipulation, vector construction, and Agrobacterium transformation were carried out according to previously published methods (Mitsuhara et al. 1996; Sambrook et al. 1998; Park 2008; Kang et al. 2012).

\section{Salt and fungal elicitation of spearmints}

The effect of salt stress on transgenic spearmints were determined by exposure of wild type and each transgenic spearmint (MSIso and MSLimo) to a high salt concentration $(800 \mathrm{mM} \mathrm{NaCl}$ ) for 3 days. After sub-culture for 4 weeks, these were transferred to salt media. The role of terpenoids in conferring protection against pathogenic fungi was determined based on resistance of transgenics to fungal infection. B. cinerea (Gray mold) and G. cingulata (Anthracnose) were used as fungal elicitors. The Fungi were cultured in YEP liquid media under dark conditions at $24 \pm 1^{\circ} \mathrm{C}$ and at $120 \mathrm{rpm}$ on rotary shaker for 1 week. After fungal culture, the fungal growth medium was filtered by sterilized $3 \mathrm{M}^{\mathrm{TM}}$ filter papers ( $3 \mathrm{M}$ co. Canada). The filtrate was inoculated such that it covered medium surface in cul- ture vessels (about $4 \mathrm{~mL}$ ) where wild type and each transgenic spearmint plant was cultured and incubated for 4 weeks. Infected plants were maintained at $25 \pm 1^{\circ} \mathrm{C}$ under $16 \mathrm{~h}$ with photoperiod for 3 days. Resistance index and elicitation were determined based on the previous study (Aoki et al. 2003; Aoki et al. 2005; Park 2008). This is a kind of morphological or observational index based on the levels of plant response after infection. The detailed resistance index was described as this (resistance index 0: Healthy shoots with all leaves green and healthy, resistance index 1: Single leaves yellowish-brown, resistance index $2:<25 \%$ of leaves yellowish-brown, resistance index 3: 26-50\% of leaves yellowish-brown, resistance index $4: 51-75 \%$ of leaves yellowish-brown, resistance index 5: 76-100\% of leaves yellowish-brown).

\section{Extraction and metabolite profiling of spearmint}

The terpenoids of spearmint for GC analysis were prepared adopting modified simultaneous steam distillation and extraction apparatus (SDE) method with Nickerson and Liken instrument (Nickerson and Likens 1996). Briefly, $10 \mathrm{~g}$ of each fresh plants and elicited plants by salt and fungi were harvested, homogenized and transferred to a steam distillation flask. Blended $25 \mathrm{~mL}$ ether and $25 \mathrm{~mL}$ n-pentane were injected into the extraction flask. Both flasks were heated on heating mantle for $1 \mathrm{hr}$. The floating organic layer of metabolites was carefully collected after enough refrigeration and using cool water. The extracts were left overnight along with sodium sulfate $\left(\mathrm{NaSO}_{4}\right)$ for dehydration. Finally each extract was evaporated in a rotary evaporator at $30^{\circ} \mathrm{C}$ under nitrogen gas $\left(\mathrm{N}_{2}\right)$.

Metabolite compositions of spearmint (MSIso and MSLimo) extracts were determined through metabolite profiling with GC-MS qualitative analysis. Each plant extract was subjected to Gas Chromatograph (HP5890 SERIES II) - Mass Spectrometer (GC-MS, HP 5971 SERIES MSD) for metabolite profiling. The GC column of chromatogram was $60 \mathrm{~mm} \times 0.25 \mathrm{~mm} \times 0.25 \mu \mathrm{m}$ i. d.HP-1 fused silica capillary column. The GC conditions were as follows: injector temperature, $250^{\circ} \mathrm{C}$; column temperature, isothermal at $50^{\circ} \mathrm{C}$ for $5 \mathrm{~min}$, then programmed to $240^{\circ} \mathrm{C}$ for $3 \mathrm{~min}$ and held at this temperature for $10 \mathrm{~min}$; ion source temperature, $230^{\circ} \mathrm{C}$. Helium was used as the carrier gas at the rate of $1 \mathrm{~mL} / \mathrm{min}$. The effluent of the $\mathrm{GC}$ 
column was introduced directly into the source of the mass spectrometer. Mass Spectra was recorded in EI mode with $70 \mathrm{eV}$ ionization energy. The sector mass analyzer was set to scan from 50 to $800 \mathrm{amu}$ in $2 \mathrm{~s}$. The identity of terpenoids was established by comparison of the retention times obtained with authentic standards. When an authentic sample was not available, the identification was carried out by comparison of mass spectra with those available in the mass spectra library (The Wiley Registry of Mass Spectral Data, $6^{\text {th }}$ ed.). The concentration of terpenoids was determined on the basis of relative area $(\%)$ of the analyzed peaks.

\section{Statistical analysis}

Resistance index was determined mean with standard deviation in three replications. Differences in the means of each value were determined by one-way ANOVA followed

Table 1. Variation in the composition (\%) of terpenoids in spearmint after salt-stress. Concentration was determined on the basis of relative percentage of peaks area on chromatography. '-' means not detected by GC/MS analysis

\begin{tabular}{|c|c|c|c|c|c|c|c|}
\hline \multirow{2}{*}{ Terpenoids } & \multirow{2}{*}{ Components } & \multicolumn{3}{|c|}{ No-Salt } & \multicolumn{3}{|c|}{ Salt } \\
\hline & & WT & MSIso & MSLimo & WT & MSIso & MSLimo \\
\hline \multirow[t]{20}{*}{ Monoterpenes } & 1,8-cineole & - & 0.25 & - & - & - & - \\
\hline & 1-a-terpineol & - & 0.2 & - & - & - & - \\
\hline & a-pinene & - & - & - & - & 0.02 & - \\
\hline & a-pinene -isomer & - & - & - & - & 0.02 & - \\
\hline & $\beta$-pinene & - & - & - & 0.02 & 0.02 & - \\
\hline & $\beta$-pinene-isomer & - & - & - & - & 0.02 & - \\
\hline & Borneol & - & 0.17 & - & - & - & - \\
\hline & Camphor & - & 0.13 & - & - & - & - \\
\hline & Carvone & - & - & - & 0.02 & 0.02 & - \\
\hline & $\gamma$-terpinene & - & - & - & 0.02 & 0.02 & 0.02 \\
\hline & Geraniol & - & 0.09 & - & - & - & - \\
\hline & Isoamyl isobutyrate & & - & - & - & - & 0.02 \\
\hline & Limonene & 0.05 & 0.1 & 0.9 & 0.02 & 0.5 & 0.59 \\
\hline & Linalool & 0.01 & 0.07 & 0.02 & - & - & - \\
\hline & Menthol & - & - & - & 0.02 & 0.02 & 0.02 \\
\hline & Menthol-isomer & - & - & 0.11 & - & - & - \\
\hline & Menthone & & - & 0.07 & 0.02 & 0.02 & - \\
\hline & Myrcene & - & - & - & - & 0.02 & - \\
\hline & $\rho$-cymene & - & - & - & 0.02 & 0.02 & 0.02 \\
\hline & Piperitone & - & - & 0.18 & - & - & \\
\hline \multirow{2}{*}{ Terpenoids } & \multirow{2}{*}{ Components } & \multicolumn{3}{|c|}{ No-Salt } & \multicolumn{3}{|c|}{ Salt } \\
\hline & & WT & MSIso & MSLimo & WT & MSIso & MSLimo \\
\hline \multirow[t]{5}{*}{ Monoterpenes } & Safranal & - & 0.49 & - & - & - & \\
\hline & Terpinene & 0.04 & 0.07 & 0.05 & - & - & \\
\hline & Terpinolene & - & - & - & - & 0.02 & \\
\hline & Thymol & 0.07 & 0.05 & 0.66 & 0.02 & 0.02 & 0.75 \\
\hline & a-cedrene & - & - & - & - & - & 0.02 \\
\hline \multirow[t]{3}{*}{ Sesquiterpenes } & a-cubebene & - & - & - & - & - & 0.02 \\
\hline & $\beta$-caryophyllene & - & - & - & - & - & 11.45 \\
\hline & Bisabolene & - & - & - & - & - & 1.78 \\
\hline \multirow[t]{3}{*}{ Phenylpropene } & Anethol & - & - & - & - & 0.02 & - \\
\hline & Eugenol & - & - & - & 0.02 & 0.02 & - \\
\hline & Methyl eugenol & - & 0.02 & - & - & - & - \\
\hline
\end{tabular}


using the Statistical Analysis System software, Version 9.0 (SAS Institute, Cary, NC, USA).

\section{Results}

\section{Variation in terpenoid composition of transgenic spearmint under salt stress}

The comparative analysis of the $M$. spicata plants experiencing salt stress revealed an increase in the number of terpenoid compounds (Table 1 and Fig. 1). In the absence of salt stress four terpenoids were detected from the spearmint extracts, all of them being monoterpenes. The transgenic
(MSIso) extracts contained eleven terpenoids (10 monoterpenes and 1 phenylpropene) while transgenic (MSLimo) extracts contained seven monoterpenes. On the other hand the extracts of plants exposed to salt-stress contained nine terpenoids that could be subdivided into seven monoterpenes and a phenylpropane.

In the absence of salt stress only four terpenoids of the monoterpene type were detected from the spearmint extracts. The salt stressed transgenic (MSIso) extracts contained fifteen terpenoids (13 monoterpenes and 2 phenylpropenes) while transgenic (MSLimo) extracts contained ten terpenoids ( 7 monoterpenes and 3 sesquiterpenes).
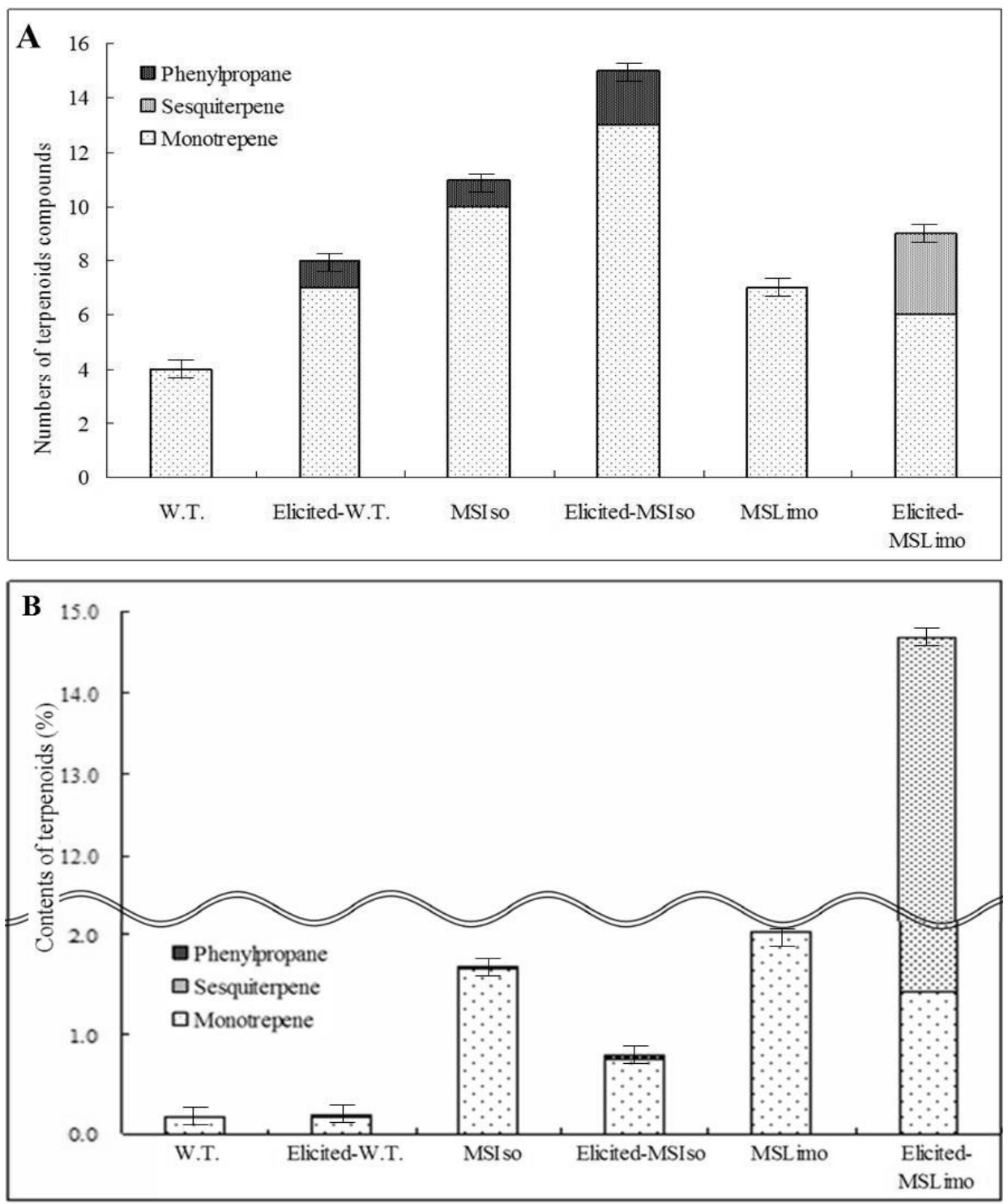

Fig. 1. Variations of terpenoid compositions in wild type and transgenic spearmints after salt stress as aboitic elicitation. (A) Number of terpenoid compounds composition between wild type and transgenic spearmints (B) Content of terpenoid (\%) compositions between wild type and transgenic spearmints. Total content of terpenoids percentages was determined on the basis of relative percentage of peaks area on chromatography. Content of terpenoids percentage provide how much monoterpenes transfer to phenyl- or sequi- terpenes during abiotic elicitation. 


\section{Variations in the composition of terpenoids in trans- genic spearmint after fungal elicitation}

The biological elicitation of wild and transgenic spearmint plants with filtrates of fungal cultures of $B$. cinerea and G. cingulata affected the metabolite composition. After 1 day of treatment with $B$. cinerea of filtrate, resistance of spearmint against $b$. cinerea are shown (Fig. 2). The resistance index of wild type was 2.66 , and transgenic plants were determined to be 1.22 (MSIso) and 0.34 (MSLimo). The wild types spearmint was damaged, but both the transgenic plants were maintained with less damage. But after 2 days following fungal treatment, the infection seemed to increase due to the fungal proliferation. After 3 days, the resistance indexes were recorded as 4.68, 4.56 and 4.34 for wild type, MSIso and MSLimo respectively. Further it was observed that the wild type plant died, but both transgenics spearmints remained alive. Among the two MSLimo plants were showed higher resistance to B. cinerea than MSIso spearmint.

Fig. 3 showed the resistance of spearmint plants to $G$. cingulata infection. After 1 day of treatment with $G$. cingulata filtrate, the damage to wild type and both transgenic plants was assessed and was shown to be similar. After 2 days of $G$. cingulata treatment, the health of spearmint plants deteriorated due to proliferation of fungus. The effects of fungal infection are expressed in terms of the resistance index. The resistance index was 2.84 for wild type and 2.72 (MSIso) and 1.21 (MSLimo) for transgenic spearmints. After 3 days, the resistance indices further increased to 4.38, 3.89 and 2.04 for wild type, MSIso and MSLimo respectively. The MSLimo plant showed highest resistance against $G$. cingulata. Furthermore, wild type spearmints were completely covered and damaged with fungal hypha of $G$. cingulata while MSIso transgenic spearmints were partially damaged from fungal infection.

\section{Discussion}

Limonene, linalool, terpinene and thynol were detected in both wild type and transgenic spearmint under control condition for all lines, but after salt treatment/elicitation only Limonene and thynol were detected among both wild type and transgenic spearmint metabolites. It seems that the production of terpenoids is obstructed by salt stress. The stress modified the production of monoterpenes like $a$ pinene, carvone, a-terpinene, menthol, myrcene, p-cymene, and thymol and phenylpropenes like anethol and eugenol appeared after elicitor exposure. It seems that these terpenoids are metabolites of spearmint against salt-stress, as the same were not detected under control conditions. Furthermore, MSIso and MSLimo transgenic spearmint extracts analyzed under non-elicited condition generally produced some additional terpenoids than those presented in wild type. Although salt stressed MSIso plant extracts showed similar metabolite profile, the salt stressed MSLimo plant extracts produced a different set of terpenoids than those presented in both transgenic lines. These results indicated that spearmint responded to salt-stress and underwent regulation of terpenoid metabolism by enhanced flux of metabolites.

a-Pinene, a-pinene-isomer, $\beta$-pinene-isomer, myrcene, terpinolene, anethol and eugenol were detected from salt stressed-MSIso plant extracts, but 1, 8-cineol, 1-alpha-terpineol, borneol, camphor, safranal and methyl eugenol disappeared. It seems that these compounds are regulated by Iso to endure salt-stress. Thus salt-stress, influenced variations in the proportions of terpenoids compounds in MSIso plant.

MSLimo plant when subjected to salt stress showed the occurrence of isoamyl isobutyrate, a-cedrene, a-cubebene, $\beta$-caryophyllene and bisabolene in their extracts, among these $\alpha$-cubebene, $\beta$-caryophyllene and bisabolene are sesquiterpenes. However the menthol-isomer, menthone and piperitone disappeared among the metabolite profile after salt stress.

Sesquiterpenes and phenylpropenes belong to larger members in the terpenoids biosynthesis pathway. These results suggested that Iso and Limo elaborate gene products in spearmint that modify the terpenoid composition by generating high-molecular weight terpenoids under salt-stress.

The purified $\alpha$ - and $\beta$-pinenes are themselves flavour and fragrance ingredients (Hall and Oser 1965). However, by far the largest uses of $\alpha$ - and $\beta$-pinene and the two enriched fractions are starting materials in the synthesis of a wide range of other flavor and fragrance ingredients, such as linalool and geraniol, and medicinal products such as vitamins $\mathrm{A}$ and $\mathrm{E}$. 
A
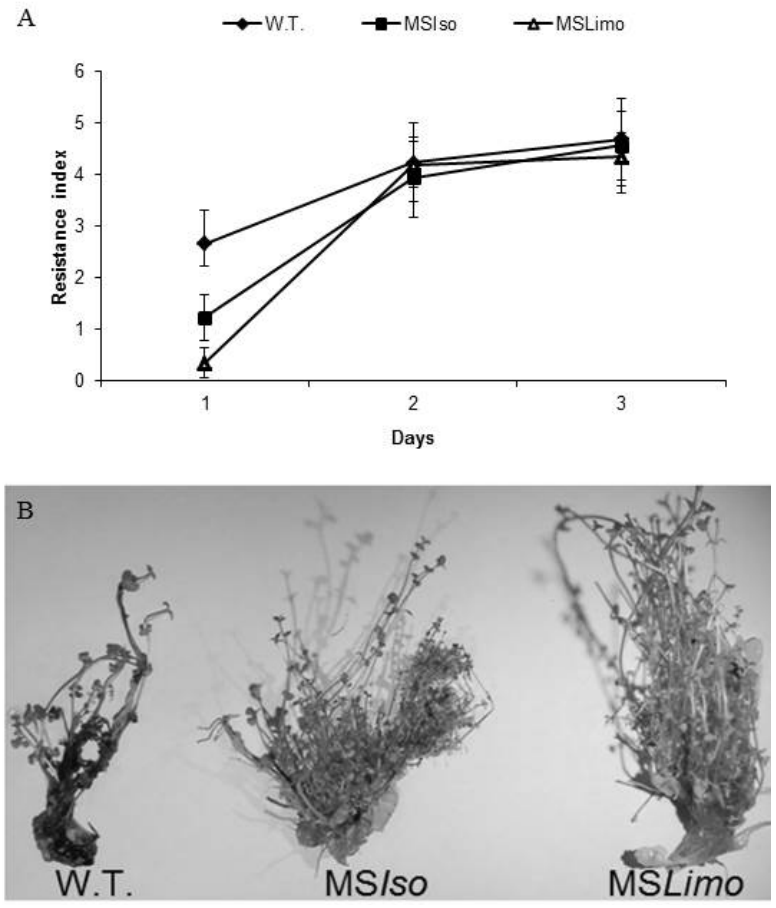

Fig. 2. Resistance of spearmint against $B$. cinerea infection. (A) Resistance index 0 : Healthy shoots with all leaves green and healthy, resistance index 1 : Single leaf yellowish-brown, resistance index $2:<25 \%$ of leaves yellowish-brown, resistance index 3:26-50\% of leaves yellowish-brown, resistance index 4: 51-75\% of leaves yellowish-brown, resistance index 5: 76-100\% of leaves yellowish-brown). (B) B. cinerea inoculated spearmint plants; wild type (W.T.), MSIso, and MSLimo plants after 3 days.

Carvone $\left(\mathrm{C}_{10} \mathrm{H}_{14} \mathrm{O}\right)$ is the main constituent of spearmint oil, and is also a constituent of other volatile oils like those from dill and caraway seed. Carvone is also an oxidation product of $d$-limonene, the main constituent of volatile oils from some citruses. Furthermore, L-carvone has also been isolated from the volatile oil of Chrysanthemum balsamita and its isomer, L-carvone, from mugwort (Artemisia vulgaris $\mathrm{L}$ ), both these being members of the Compositae family. Due to its spearmint-like odour, carvone is used as a flavouring in many products, such as candies, chewing gums, mouthwashes and toothpastes (Corazza et al. 2002). Myrcene is a naturally occurring acyclic polyunsaturated monoterpene which contains three carbon-carbon double bonds, two of them being conjugated. This monoterpene is also easily available by the industrial pyrolysis of $\beta$-pinene, one of the major constituents of pine turpentine (Erman 1985; Lawrence 1989). p-Cymene is also a constituent of oregano and thyme oils but is less effective against food-re-
A
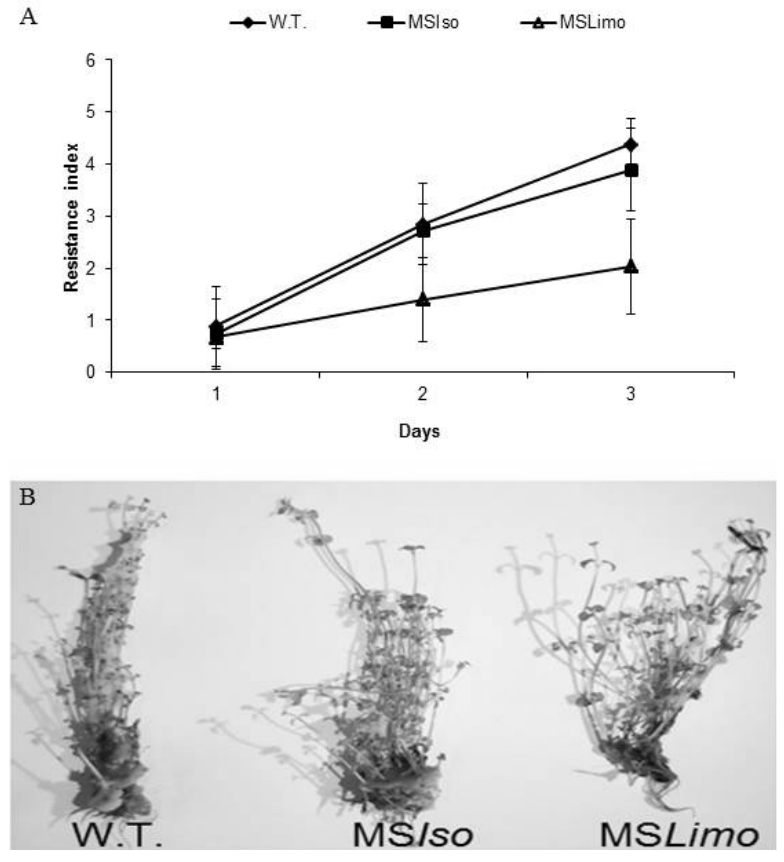

Fig. 3. Resistance of spearmints against $G$. cingulata infection. (A) Rresistance index ( 0 : Healthy shoots, all leaves green and healthy; 1 : Single leaf yellowish-brown; 2: $<25 \%$ of leaves yellowish-brown; $3: 26-50 \%$ of leaves yellowish-brown; 4: 51-75\% of leaves yellowish-brown; 5: 76-100\% of leaves yellowish-brown). (B) G. cingulata treated wild type plant, MSIso plant, MSLimo plants after 3 days.

lated pathogens (Dorman and Deans 2000; Ultee et al. 2000; Burt et al. 2005) and is thought to be a precursor to carvacrol and thymol in the plant (Ultee et al. 2002).

Terpinolene is a monoterpenic constituent of some essential oils of various fir and pine species, as well as plants such as Manilla elemi, Nectranda elaiophora, and Dacrydium colensoi (Burdock 1995). It displays antifungal activity against various pathogens (Himejima et al. 1992). Hence, it seemed interesting to study the detoxification of antifungal monoterpene terpinolene by plant pathogenic fungus Botris cinerea as an exemplary eco-chemical interaction of the aromatic plants and phytopathogenic fungi. a-Cedrene is a major component of cedar wood oil which is used widely as a perfume and as a precursor of synthetic perfumes for toiletry and cosmetic products. Abraham and coworkers reported that Corynespora cassicola DSM 62474 and Rhodococcus rhodochrous (formerly Mycobacterium rhodochrous) ATCC 999 converted a-cedrene to a variety of minor products, such as 3-hydroxy- and 12-hydroxy-a-cedrenes (Abraham et al. 1987) and cedrenone and 10-methoxy-a-ce- 
drene, respectively (Kieslich et al. 1986). The formation of such products from a-cedrene by Beauveria sulfurescens ATCC 7159 was also reported (Lamare et al. 1987; Lamare and Furstoss 1990) However, the biotransformations of a-cedrene reported thus far require a long cultivation time, and the yields of products have been extremely low.

a-Cubebene is the precursor for calamenene via one of two pathways. One path requires the conversion of a-cubebene to (-)cubenol, to cadina-4,6(1) diene and then to calamenene (Menary et al. 1999). B-Caryophyllene, is a natural bicyclic sesquiterpene with a rare cyclobutane ring. $\beta$ caryophyllene is known for its anti-inflammatory and local anaesthetic activities (Tambe et al. 1996; Ghelardini et al. 2001). It is used in spice blends, citrus flavors, soaps, detergents, creams and lotions, and also in a variety of food products and beverages (Budavari 1996; Sköld et al. 2006). It has been reported as a volatile compound emitted by plants into the atmosphere in response to herbivore attack and due to change in abiotic factors (Gouinguené and Turlings 2002) and as sequestered by the Australian green tree frog, Litoria caerulea White from its diet (Smith et al. 2004).

Anethol is the main antimicrobial active molecule of anise oil. In general, the antimicrobial activity of anethol has been related to the ether group on its aromatic ring (Davidson and Naidu 2000). Although anethol had a high capacity to modify rumen microbial fermentation, it reduced the proportion of acetate and propionate, which are the main precursors in ruminants for fat and glucose synthesis, respectively, which suggests that anethol may not be nutritionally beneficial to dairy cattle. Eugenol is natural flavor extracts from plants. Eugenol is used as the substrate of bioconversion. Eugenol is converted under strong basic condition to isoeugenol that was subsequently transformed to vanillin by Lipoxygenase (Li et al. 2004).

In conclusion, the secondary metabolites like terpenoids increase following fungal and salt stress as biotic and abiotic elicitors. The metabolically engineered transgenic spearmint with MSIso and MSLimo proved beneficial for general enhancements of terpenoids and their yields. The efforts undertaking the investigations on overproduction of secondary metabolites from transgenic spearmint by employing elicitation are scarce. Such an attempt offers a dou- ble advantage for enhanced production of metabolites like terpenoids. The elicitors like fungi and salt enhanced the diversity of terpenoids in MSIso and MSLimo transgenic spearmints. Hence, the combination of genetic modification and elicitation experiments could contribute towards developing novel production technologies for enhancing the ever increasing demand for essential oils by reducing time-related processes in forest biotechnology.

\section{Acknowledgements}

This work was mainly supported by Forest Science \& Technology Projects (Project No.S211010L20120) by Korea Forest Service. Additionally, this work was also partially supported by the Construction of the Basis for Practical Application of Herbal Resources (K13020) \& Development of Basic Technologies for the Domestic Cultivation of Herbal Medicine Resources (K14417), the Korea Institute of Oriental Medicine (KIOM) to the Ministry of Science, ICT \& Future Planning (MSIP), and Republic of Korea.

\section{References}

Abraham WR, Washausen P, Wieslich KK. 1987. Microbial hydroxylation of cedrol and cedrene. Z Naturforsch 42C: 414-419.

Aoki T, O'Donnell K, Homma Y, Lattanzi AR. 2003. Suddendeath syndrome of soybean is caused by two morphologically and phylogenetically distinct species within the Fusarium solani species complex--F. virguliforme in North America and F. tucumaniae in South America. Mycologia 95: 660-684.

Aoki T, O’Donnell K, Scandiani MM. 2005. Sudden death syndrome of soybean in South America is caused by four species of Fusarium: Fusarium brasiliense sp. nov., F. cuneirostrum sp. nov., F. tucumaniae, and F. virguliforme. Mycoscience 46: 162-183.

Bienvenu F, Peterson L, Edwards J. 1999. Native and Scotch Spearmint Oil Production in Tasmania and Victoria. A report for the Rural Industries Research and Development Corporation, pp 99-147.

Bryant JP, Julkunen-Tiitto R. 1995. Ontogenic development of chemical defense by seedling resin birch: Energy cost of defense production. J Chem Ecol 21: 883-896.

Bryant JP, Provenza FD, Pastor J, Reichardt PB, Clausen TP, du Toit JT. 1991. Interactions between woody plants and browsing mammals mediated by secondary metabolites. Annu Rev Ecol Syst 22: 431-446.

Budavari S. 1996. The Merck index: an encyclopedia of chemicals, drugs, and biologicals. 12th ed. Merck \& Co Inc., New Jersey, USA. 
Burdock GA. 1995. Fenaroli's Hanbook of Flavor In-gredients: Adapted from the Italian Language Works of Giovanni Fenaroli. 3rd ed. CRC Press, Boca Raton, USA.

Burt SA, Vlielander R, Haagsman HP, Veldhuizen EJ. 2005. Increase in activity of essential oil components carvacrol and thymol against Escherichia coli O157:H7 by addition of food stabilizers. J Food Prot 68: 919-926.

Choi MS, Heu S, Paek NC, Koh HJ, Lee JS, Oh CS. 2012. Expression of hpal gene encoding a bacterial harpin protein in Xanthomonas oryzae pv. oryzae enhances disease resistance to both fungal and bacterial pathogens in rice and arabidopsis. Plant Pathology J 28: 364-372.

Corazza M, Levratti A, Virgili A. 2002. Allergic contact cheilitis due to carvone in toothpastes. Contact Dermatitis 46: 366-367.

Davidson PM, Naidu AS. 2000. Phyto-phenols. In: Natural Food Antimicrobial Systems (Naidu AS, ed). CRC Press, Boca Raton, Florida, USA, pp 265-293.

Dorman HJ, Deans SG. 2000. Antimicrobial agents from plants: antibacterial activity of plant volatile oils. J Appl Microbiol 88: 308-316.

Erman WF. 1985. Chemistry of the monoterpenes: an encyclopedic handbook. Marcel Dekker, New York.

Ghelardini C, Galeotti N, Di Cesare Mannelli L, Mazzanti G, Bartolini A. 2001. Local anaesthetic activity of beta-caryophyllene. Farmaco 56: 387-389.

Gouinguené SP, Turlings TC. 2002. The effects of abiotic factors on induced volatile emissions in corn plants. Plant Physiol 129: 1296-1307.

Hall RL, Oser BL. 1965. Recent progress in the consideration of flavoring ingredients under the food additives amendment. III GRAS Substances. Food Technol 19: 151-197.

Himejima M, Hobson KR, Otsuka T, Wood DL, Kubo I. 1992. Antimicrobial terpenes from oleoresin of ponderosa pine tree Pinus ponderosa: A defense mechanism against microbial invasion. J Chem Ecol 18: 1809-1818.

Julkunen-Tiitto R, Bryant JP, Kuropat P, Roininen H. 1995. Slight tissue wounding fails to induce consistent chemical defense in three willow (Salix spp.) clones. Oecologia 101: 467-471.

Jung HY, Kang SM, Kang YM, Kang MJ, Yun DJ, Bahk JD, Yang JK, Choi MS. 2003. Enhanced production of scopolamine by bacterial elicitors in adventitious hairy root cultures of Scopolia parviflora. Enzyme Microb Tech 33: 987-990.

Kang YM, Park DJ, Song HJ, Ma HS, Karigar C, Choi M. 2012. Comparative analysis of terpenoids in in vitro culture media of metabolically engineered transgenic and wild type spearmint (Mentha spicata L.). Korean J Medicinal Crop Sci 20: 301-307.

Kieslich K, Abraham WR, Stumpf B, Thede B, Washausen P. 1986. Transformations of terpenoids, Walter de Gruyter and Co, Berlin, Germany.

Laitinen ML, Julkunen-Tiitto R, Rousi M. 2002. Foliar phenolic composition of European white birch during bud unfolding and leaf development. Physiol Plant 114: 450-460.
Laitinen ML, Julkunen-Tiitto R, Yamaji K, Heinonen J, Rousi M. 2004. Variation in birch bark secondary chemistry between and within clones: implications for herbivory by hares. Oikos 104: 316-326.

Lamare V, Fourneron JD, Furstoss R, Ehret C, Corbier B. 1987. Microbial transformations. IX: Biohydroxylation of alpha-cedrene and cedrol. Synthesis of an odoriferous minor component of cedar wood essential oil. Tetrahedron Lett 28: 6269-6272.

Lamare V, Furstoss R. 1990. Bioconversion of sesquiterpenes. Tetrahedron 46: 4109-4132.

Lange BM, Mahmoud SS, Wildung MR, Turner GW, Davis EM, Lange I, Baker RC, Boydston RA, Croteau RB. 2011. Improving peppermint essential oil yield and composition by metabolic engineering. Proc Natl Acad Sci USA 108: 16944-16949.

Lawrence RV. 1989. Processing pine gum into turpentine and rosin. Pulp Chemicals Association, New York, USA.

Lee DW, Seo JB, Kang JS, Koh SH, Lee SH, Koh YH. 2012. Identification and Characterization of Expansins from Bursaphelenchus xylophilus (Nematoda: Aphelenchoididae). Plant Pathology J 28: 409-417.

Li YH, Sun ZH, Zheng P. 2004. Determination of Vanillin, Eugenol and Isoeugenol by RP-HPLC. Chromatographia 60: 709-713.

Menary RC, Dragar VA, Garland SM. 1999. Tasmannia lanceolata: developing a new commercial flavour product: a report for the Rural Industries Research and Development Corporation, USA.

Mitsuhara I, Ugaki M, Hirochika H, Ohshima M, Murakami T, Gotoh Y, Katayose Y, Nakamura S, Honkura R, Nishimiya S, Ueno K, Mochizuki A, Tanimoto H, Tsugawa H, Otsuki Y, Ohashi Y. 1996. Efficient promoter cassettes for enhanced expression of foreign genes in dicotyledonous and monocotyledonous plants. Plant Cell Physiol 37: 49-59.

Nickerson GB, Likens ST. 1996. Gas chromatographic evidence for the occurrence of hop oil components in beer. J Chromatogr 21: $1-5$.

Park DJ. 2008. Metabolites profiling and its function through terpenoids biosynthesis genes overexpression of spearmint (MenthaspicataL.). Master thesis. Gyeongsang National University, Jinju, Korea.

Sambrook J, Fritch EF, Maniatis T. 1998. Molecular cloning. In: A laboratory manual. Cold Spring Harbor Lab, Cold Spring Harbor, New York.

Seigler DS. 1998. Plant Seconday Metabolism. Kluwer Academic Publishers, Dordrecht, The Netherlands.

Singh HP, Batisha DR, Kohlia RK. 1999. Autotoxicity: concept, organisms, and ecological significance. CR Rev Plant Sci 18: 757-772.

Sköld M, Karlberg AT, Matura M, Börje A. 2006. The fragrance chemical beta-caryophyllene-air oxidation and skin sensitization. Food Chem Toxicol 44: 538-545. 
Smith BPC, Hayasaka Y, Tyler MJ, Williams BD. 2004. ß-caryophyllene in the skin secretion of the Australian green tree frog, Litoria caerulea: an investigation of dietary sources. Aust J Zool 52: 521-530.

Tambe Y, Tsujiuchi H, Honda G, Ikeshiro Y, Tanaka S. 1996. Gastric cytoprotection of the non-steroidal anti-inflammatory sesquiterpene, beta-caryophyllene. Planta Med 62: 469-470.

Tegelberg R, Julkunen-Tiitto R. 2001. Quantitative changes in secondary metabolites of dark-leaved willow (Salix myrsinifolia) exposed to enhanced ultraviolet-B radiation. Physiologia Plantarum 113: 541-547.

Tegelberg R , Julkunen-Tiitto R, Aphalo PJ. 2001. The effects of long-term elevated UV-B on the growth and phenolics of field-grown silver birch (Betula pendula). Global Change Biology 7: 839-848.

Ultee A, Bennik MH, Moezelaar R. 2002. The phenolic hydroxyl group of carvacrol is essential for action against the food-borne pathogen Bacillus cereus. Appl Environ Microbiol 68: 1561-1568.

Ultee A, Slump RA, Steging G, Smid EJ. 2000. Antimicrobial activity of carvacrol toward Bacillus cereus on rice. J Food Prot 63: 620-624.

Waterman PG, Mole S. 1994. Analysis of phenolic plant metabolites. Blackwell Scientific Publications, Oxford, UK. 